Artículo científico

Volumen 31(1):157-176. Enero-abril, 2020

e-ISSN 2215-3608, doi:10.15517/am.v31i1.34739

http://www.revistas.ucr.ac.cr/index.php/agromeso

\title{
Condiciones climáticas y la producción láctea del ganado jersey en dos pisos altitudinales ${ }^{1}$
}

\section{Climatic conditions and jersey cattle dairy production on two altitudinal levels}

\author{
Juan Federico Conejo-Morales ${ }^{2}$, Rodolfo WingChing-Jones ${ }^{3}$
}

1 Recepción: 20 de marzo, 2019. Aceptación: 29 de agosto, 2019. Este trabajo formó parte del trabajo de licenciatura en Ingeniería Agronómica con énfasis en Zootecnia del primer autor, financiada por la Universidad de Costa Rica, San José, Costa Rica.

2 Cuidad de los Niños, Cartago, Costa Rica. federicoconejo@gmail.com

3 Universidad de Costa Rica, Escuela de Zootecnia, Centro de Investigación en Nutrición Animal, San José, Costa Rica. rodolfo.wingching@ ucr.ac.cr (https://orcid.org/0000-0002-8009-2210).

\section{Resumen}

Introducción. La precipitación, temperatura, radiación y humedad relativa varían según la altura, esto provoca la presencia de microclimas que afectan la productividad de los animales. Objetivo. Asociar las condiciones climáticas presentes en el sistema productivo con la producción láctea de un hato jersey puro en dos pisos altitudinales de la provincia de Cartago, Costa Rica. Materiales y métodos. De forma retrospectiva, durante el año 2016, se evaluó la información productiva de dos sistemas ubicados a 660 y $1800 \mathrm{msnm}$. Del primero se analizaron los registros de 61 animales durante doce años (12 083 datos); mientras que en el segundo se analizaron las lactancias de 387 animales en cinco años (13 820 registros). Las observaciones incluyeron: identificación del semoviente, día de generación del registro, mes, año, producción de leche diaria, días de lactancia, número de partos, edad del animal, temperatura, precipitación, radiación solar y humedad relativa promedio. Resultados. Los días de lactancia, el número de lactancia (3a-5a), la edad del animal (3,7 a 5,8 años) y el número de partos afectaron la producción de leche diaria en ambas fincas (p<0,001). A $1800 \mathrm{~m}$, cuando la precipitación fue mayor a 5 pero menor o igual a $10 \mathrm{~mm} \mathrm{día}^{-1}$ y la humedad relativa mayor a 80 pero menor a $90 \%$ día $^{-1}(\mathrm{p}<0,001)$, la producción de leche disminuyó. En cambio, a $660 \mathrm{~m}$ el efecto provino de la humedad relativa menor o igual a $70 \%$. La temperatura ambiental tuvo un efecto significativo sobre ambas altitudes. Conclusiones. Las condiciones de temperatura, humedad, precipitación y radiación en cada ecosistema, generaron diferencias en la productividad de los animales, relacionadas con el tipo de forraje y la sensación térmica a la que estaba expuesto el ganado, lo cual provocó estrés por calor o por frío.

Palaras clave: Bos taurus, microclima, estrés calórico, índice de temperatura y humedad, curva de lactancia.

\begin{abstract}
Introduction. Precipitation, temperature, radiation, and relative humidity vary according to height, this causes the presence of microclimates that affect the productivity of the animals. Objective. Associate the climatic conditions present in the productive systems with dairy production of a pure Jersey herd in two altitudinal floors of the province of Cartago, Costa Rica. Materials and methods. In retrospect, during the year 2016, the productive information of two dairy farms, located at 660 and 1800 m.a.s.l. was evaluated. From the first farm, the records of 61 animals in
\end{abstract}


a twelve-year period (12,083 data) were analyzed; while in the second, the lactations of 387 animals in a five-year period $(13,820$ records) were analyzed. Observations included: identification of the cow, day of generation of the record, month, year, daily milk production, lactation days, calving number, animal age, temperature, precipitation, solar radiation and average relative humidity. Results. The lactation days, the lactation number (3-5), the age of the animal (3.7 to 5.8 years), and the calving number affected the daily milk production on both farms (p<0.001). At $1800 \mathrm{~m}$, when the precipitation was greater than 5 but less than or equal to $10 \mathrm{~mm} \mathrm{day}^{-1}$ and the relative humidity greater than 80 but less than $90 \%$ day $^{-1}(\mathrm{p}<0.001)$, milk production decreased. On the other hand, at $660 \mathrm{~m}$ the effect came from relative humidity less than or equal to $70 \%$. The environmental temperature had a significant effect in both altitudes. Conclusion. The conditions of temperature, humidity, precipitation, and radiation in each ecosystem, generated differences in the productivity of the animals, related to the type of forages and the thermal sensation to which the cattle was exposed, which caused heat or cold stress.

Keywords: Bos taurus, microclimate, heat stress, humidity and temperature index, lactation curve.

\section{Introducción}

Según el VI Censo Nacional Agropecuario elaborado por el Instituto Nacional de Estadística y Censos en el 2014 (INEC, 2015), en Costa Rica existían 37171 fincas ganaderas, con un hato total de 1278817 animales, de los cuales 327130 (25,58\% del total) correspondían a ganado lechero especializado, distribuidos en diferentes partes del país. Las razas de mayor importancia son la holstein y la jersey, que en conjunto representan el $80 \%$ del inventario nacional (Rodríguez et al., 2007), y se encuentran concentradas como razas puras en la Región Central, donde las condiciones climáticas son propicias para explotar su potencial genético (Zúñiga et al., 2005).

Costa Rica se caracteriza por presentar siete regiones climáticas, producto de dos regímenes de precipitaciones, la altura y orientación de las montañas, los vientos predominantes, la influencia del océano Pacífico y el mar Caribe. Estas regiones presentan condiciones de suelo, vegetación, temperatura, precipitación y radiación diferentes entre sí (MINAET/IMN/PNUD/CRRH, 2008); condiciones que podrían generar efectos sobre los animales, principialmente, cuando superan los límites máximos $\left(25^{\circ} \mathrm{C}\right)$ y mínimos $\left(5^{\circ} \mathrm{C}\right)$ de la zona de termo neutralidad (Kadzere et al., 2002).

El clima o microclimas presentes en cada región inciden sobre la actividad ganadera en forma directa, debido a que actúan sobre la fisiología productiva del animal (Berman, 2011). De forma indirecta afecta la productividad (Pezzopane et al., 2016) y calidad del forraje (Sánchez et al., 2000), la fluctuación de las poblaciones parasitarias (Jiménez et al., 2007), el microambiente de los establecimientos de resguardo, el almacenamiento de alimentos y el proceso de mercadeo de productos (Retana, 2015). De forma directa la temperatura ambiental, la humedad relativa, la radiación solar, la velocidad del viento, el efecto de la duración del día y la precipitación diaria afectan la productividad láctea de los animales (WingChing-Jones et al., 2008). Por otro lado, el índice de temperatura y humedad (IHT), es un buen indicador para estimar condiciones de estrés en vacas en condiciones subtropicales (Dikmen y Hansen, 2009).

Estos factores afectan al ganado al reducirle el aporte de nutrimentos vía ingesta de alimento (West, 2003), además altera las concentraciones hormonales (Jordan, 2003), el intercambio de calor (Collier et al., 2006) y el balance energético de los animales (Khalifa, 2003), lo que provoca que los animales deban usar los nutrimentos requeridos para mantenimiento, crecimiento, producción y reproducción (NRC, 2001), en el control de la temperatura corporal, así como en mecanismos fisiológicos para mantener la termoneutralidad (Collier et al., 2003) y la salud.

Este trabajo tuvo como objetivo asociar las condiciones climáticas presentes en cada sistema productivo con la producción láctea de un hato jersey en dos pisos altitudinales de la provincia de Cartago, Costa Rica. 


\section{Materiales y métodos}

\section{Características del estudio}

Esta investigación consistió en el análisis retrospectivo de las condiciones climáticas registradas en dos sistemas de producción de ganado jersey, ubicado en dos pisos altitudinales y su relación con la producción láctea de los animales. Además, exploró el comportamiento de la productividad de los animales, el número de lactancia y los días de lactancia en el caso de variables intrínsecas de los semovientes. En el caso de las variables temporales, se exploró el efecto del año y el mes sobre la producción láctea.

\section{Localización y caracterización agroecológica de los sistemas de producción}

La investigación se realizó en la provincia de Cartago Costa Rica, en las localidades de Santa Rosa (1800 msnm) y en Turrialba (660 msnm). Santa Rosa es un distrito del cantón de Oreamuno, Cartago, que se ubica en la sección oriental del Valle Central (latitud $09^{\circ} 54^{\prime}$, longitud 8349'). Esta región se caracteriza por tener influencia climática del Caribe y poseer bosque húmedo tropical premontano, alta humedad, presencia de neblina y bosque primario y siempreverde, de dos estratos (Quesada, 2007). El segundo sistema de producción, se encuentra en Turrialba, en la Sede del Atlántico de la Universidad de Costa Rica, denominada Módulo Lechero; unidad dedicada a la investigación, la acción social y la docencia de dicha institución. Se ubica en el distrito central (latitud $09^{\circ} 88^{\prime}$, longitud $83^{\circ} 60^{\prime}$ ), posee clima lluvioso, con bosque muy húmedo subtropical de media altura siempreverde, de dos o tres estratos (Quesada, 2007). En el Cuadro 1, se resumen las características agroecológicas y de manejo promedio que se realizaron en los sistemas de producción, donde se recopiló, ordenó y analizó la información durante el año 2016.

Cuadro 1. Condiciones agroecológicas presentes en Santa Rosa de Oreamuno y Turrialba durante el periodo de la investigación. Cartago, Costa Rica. 2016.

Table 1. Agroecological conditions present in Santa Rosa of Oreamuno and Turrialba during the research period. Cartago, Costa Rica. 2016.

\begin{tabular}{|c|c|c|}
\hline Variable & Santa Rosa de Oreamuno & Turrialba \\
\hline \multicolumn{3}{|l|}{ Condiciones agroecológicas } \\
\hline Precipitación (mm) & 2239 & 2662 \\
\hline Radiación (h día ${ }^{-1}$ ) & 5,5 & 17,4 \\
\hline Altura (msnm) & 1800 & 660 \\
\hline Temperatura mínima $\left({ }^{\circ} \mathrm{C}\right)$ & 13,4 & 17 \\
\hline Temperatura máxima $\left({ }^{\circ} \mathrm{C}\right)$ & 23,3 & 24 \\
\hline Velocidad del viento $\left(\mathrm{km} \mathrm{h}^{-1}\right)$ & 24,4 & 1,8 \\
\hline \multicolumn{3}{|l|}{ Sistema de alimentación } \\
\hline Tipo de forraje & Kikuyo (Kikuyocloa clandestina) & Estrella (Cynodonnlemfluensis) \\
\hline Rotación de potreros (días) & 37 & 25 \\
\hline Suplementación en canoa & Sí & No \\
\hline Hectáreas para animales en producción & 35 & 4 \\
\hline Total de animales & 240 & 40 \\
\hline Animales en ordeño & 180 & 16 \\
\hline Promedio de producción $\left(1\right.$ animal $\left.{ }^{-1}\right)$ & 18 & 15 \\
\hline Litros de leche $\left(\right.$ día ha $\left.{ }^{-1}\right)$ & 50 & 55,5 \\
\hline
\end{tabular}




\section{Información meteorológica utilizada en la investigación}

Se utilizaron los datos obtenidos de dos estaciones meteorológicas, la primera ubicada en el Centro Agronómico Tropical de Investigación y de Enseñanza (CATIE), situado a 1 km de distancia del Módulo Lechero, y la segunda, ubicada en la propia finca en Santa Rosa. En ambos sistemas, las estaciones meteorológicas utilizadas registraron de forma diaria el valor promedio de la temperatura máxima y mínima $\left({ }^{\circ} \mathrm{C}\right)$, la precipitación $(\mathrm{mm})$, la radiación solar $\left(\mathrm{MJ} \mathrm{m}^{-2}\right)$ y la humedad relativa máxima y mínima $(\%)$.

\section{Período de evaluación y de recolección de datos}

La información base para este estudio comprende los datos meteorológicos y productivos recolectados desde el año 2003 al 2014 para la finca que se encuentra a 660 msnm, y desde el año 2011 al 2015 para el caso de Santa Rosa. Se analizó la información de 61 animales para el sistema a $660 \mathrm{msnm}$ durante doce años y se obtuvieron 12089 observaciones. En cuanto al sistema a $1800 \mathrm{msnm}$, se analizaron 387 animales y se obtuvieron 13820 observaciones en cinco años. Tal diferencia en los períodos de recolección de datos se sustenta en el número de registros evaluados, pues para cada registro adquirido en el sistema de producción en Turrialba, se obtuvieron seis en el sistema productivo de Santa Rosa.

\section{Organización de la información recolectada por sistema}

Para la organización de la información se confeccionó una base de datos en una hoja de cálculo para cada sistema de producción, donde se digitó la información de la siguiente manera: identificación del animal, mes de la lectura, año de la lectura, edad del semoviente (meses), número de lactancia, producción diaria de leche (kg día $\mathrm{a}^{-1}$ ), días de lactancia, temperatura $\left({ }^{\circ} \mathrm{C}\right)$, precipitación $(\mathrm{mm})$, radiación $\left(\mathrm{MJ} \mathrm{m}^{-2}\right)$, humedad relativa $(\%)$ índice de humedad y temperatura (IHT). En el caso del sistema de producción ubicado a $1800 \mathrm{msnm}$, no se tuvo acceso a la información de la edad de los animales, por lo que solo para la finca ubicada a $660 \mathrm{msnm}$ se le realizó el análisis respectivo de la edad del animal y la productividad del animal. La determinación del índice de humedad y temperatura, se realizó mediante la ecuación descrita por Martínez (2011) (ecuación 1), donde TA = temperatura ambiente y HR = humedad relativa.

$$
\mathrm{ITH}=0,81 * \mathrm{TA}+(\mathrm{TA}-14,4) *(\mathrm{HR} / 100)+46,4 \quad \text { (ecuación 1) }
$$

En los Cuadros 2 y 3 se describen las variables evaluadas en esta investigación, consideradas como temporales (año y mes), intrínsecas del animal y ambientales, de igual manera, se describen las medidas de dispersión que se encontraron para cada variable, como lo son el promedio, la desviación estándar y los valores máximos y mínimos utilizados.

\section{Curva de lactancia y persistencia}

Para generar la curva de lactancia con la información recopilada, se procedió a agrupar la información según rangos en días de lactancia, para el periodo de 0 a 200 días se trabajó con agrupamientos de 15 días, lo cual permitió obtener 12 puntos de la curva, entre los 200 y 500 días los agrupamientos fueron cada 50 días lo que permitió 7 puntos de la curva, para un total de 19 puntos. En el caso de la persistencia, posterior a organizar los puntos y visualizar el rango de días que permitió obtener la mayor producción, se calculó como el porcentaje de disminución que presentó el grupo de datos con respecto al siguiente, de mayores días de lactancia. 
Cuadro 2. Condiciones ambientales e indicadores productivos utilizados en el sistema de producción láctea de un hato jersey, ubicado a 660 msnm. Turrialba, Cartago, Costa Rica. 2016.

Table 2. Environmental conditions and productive indicators used in the milk production system of a jersey herd located at 660 m.a.s.1. Turrialba, Cartago, Costa Rica. 2016.

\begin{tabular}{|c|c|c|c|c|c|}
\hline Variables* & Unidades & Valor promedio & $\begin{array}{l}\text { Desviación } \\
\text { estándar }\end{array}$ & Valor máximo & Valor mínimo \\
\hline \multicolumn{6}{|l|}{ Temporales } \\
\hline Año & Años & - & - & 2014 & 2003 \\
\hline Mes & Meses & - & - & 12 & 1 \\
\hline \multicolumn{6}{|l|}{ Intrínsecas } \\
\hline Lactación & Cantidad & 2,97 & 1,96 & 10 & 1 \\
\hline Edad & Meses & 61,72 & 27,12 & 145 & 20 \\
\hline Kilos de leche & kg día ${ }^{-1}$ & 15,39 & 4,11 & 32,60 & 3,0 \\
\hline Días en lactancia & Días & 183,25 & 122,42 & 670 & 1 \\
\hline \multicolumn{6}{|l|}{ Ambientales } \\
\hline Temperatura & ${ }^{\circ} \mathrm{C}$ & 22,27 & 1,30 & 26,20 & 17,10 \\
\hline Precipitación & mm día ${ }^{-1}$ & 7,65 & 19,01 & 429 & 0 \\
\hline Radiación & $\mathrm{MJ} \mathrm{m}^{-2}$ & 15,98 & 5,80 & 27,70 & 0 \\
\hline Humedad relativa & $\%$ & 90,56 & 5,84 & 100 & 4,30 \\
\hline $\mathrm{IHT}^{* *}$ & & 71,54 & 2,18 & 76,60 & 61,60 \\
\hline
\end{tabular}

* Los resultados obtenidos para cada variable provienen del análisis de una base de datos de 12089 observaciones / The results obtained for each variable come from the data base analysis of 12089 observations.

**Índice humedad y temperatura / temperature-humidity index (THI).

Cuadro 3. Condiciones ambientales e indicadores productivos utilizados en el sistema de producción láctea de un hato jersey, ubicado a 1800 msnm. Santa Rosa de Oreamuno, Cartago, Costa Rica. 2016.

Table 3. Environmental conditions and productive indicators used in the milk production system of a jersey herd located at $1800 \mathrm{~m} . \mathrm{a} . \mathrm{s} .1$. Santa Rosa of Oreamuno, Cartago, Costa Rica. 2016.

\begin{tabular}{|c|c|c|c|c|c|c|}
\hline Variables & $\begin{array}{l}\text { Número observa- } \\
\text { ciones evaluadas }\end{array}$ & Unidades & Valor promedio & $\begin{array}{l}\text { Desviación } \\
\text { estándar }\end{array}$ & Valor máximo & Valor mínimo \\
\hline \multicolumn{7}{|l|}{ Temporales } \\
\hline Año & 14641 & Años & - & - & 2015 & 2011 \\
\hline Mes & 14641 & Meses & - & - & 12 & 1 \\
\hline \multicolumn{7}{|l|}{ Intrínsecas } \\
\hline Lactación & 14641 & Cantidad & 3,13 & 2,04 & 11 & 1 \\
\hline Kilos de leche & 13820 & kg día ${ }^{-1}$ & 20,02 & 4,93 & 42 & 2 \\
\hline Días de lactación & 14639 & Días & 138,64 & 87,21 & 558 & 10 \\
\hline \multicolumn{7}{|l|}{ Ambientales } \\
\hline Temperatura & 13722 & ${ }^{\circ} \mathrm{C}$ & 15,09 & 1,17 & 17,70 & 11,60 \\
\hline Precipitación & 13722 & mm día ${ }^{-1}$ & 1,97 & 4,53 & 28,40 & 0 \\
\hline Radiación & 13723 & $\mathrm{MJ} \mathrm{m}^{-2}$ & 20,85 & 11,94 & 60,70 & 0 \\
\hline Humedad relativa & 13722 & $\%$ & 80,73 & 9,97 & 99,40 & 52 \\
\hline $\mathrm{ITH}^{*}$ & 13722 & & 59,34 & 1,88 & 63,10 & 53,20 \\
\hline
\end{tabular}

*Índice temperatura y humedad / Temperature-humidity index (THI). 


\section{Variables ambientales y producción láctea}

A la hora de relacionar las variables ambientales con la producción de leche, se procedió a generar rangos con base en la dispersión de los datos en cada variable (Cuadro 4).

Cuadro 4. Organización de las variables ambientales y la edad de los animales según la ubicación del sistema de producción láctea. Cartago, Costa Rica. 2016.

Table 4. Organization of the environmental variables and the age of the animals according to the location of the milk production system. Cartago, Costa Rica. 2016.

\begin{tabular}{|c|c|c|}
\hline \multirow[t]{2}{*}{ Variables } & \multicolumn{2}{|c|}{ Sistemas de producción } \\
\hline & $660 \mathrm{msnm}$ & $1800 \mathrm{msnm}$ \\
\hline Radiación (MJ día ${ }^{-1}$ ) & $\begin{array}{c}\leq 10 \\
>10,001 \leq 20 \\
>20,001\end{array}$ & $\begin{array}{c}\leq 10 \\
>10,001 \leq 25 \\
>25,001 \leq 35 \\
>35,001 \leq 45 \\
>45,001\end{array}$ \\
\hline Humedad relativa $(\%)$ & $\begin{array}{c}\leq 70 \\
>70,001 \leq 80 \\
>80,001 \leq 90 \\
>90,001\end{array}$ & $\begin{array}{c}\leq 70 \\
>70,001 \leq 80 \\
>80,001 \leq 90 \\
>90,001\end{array}$ \\
\hline Temperatura $\left({ }^{\circ} \mathrm{C}\right)$ & $\begin{array}{c}\leq 20 \\
>20,001 \leq 23 \\
>23,001\end{array}$ & $\begin{array}{c}\leq 14 \\
>14,001 \leq 16 \\
>16,001 \\
\end{array}$ \\
\hline Precipitación (mm día $\left.{ }^{-1}\right)$ & $\begin{array}{c}\leq 40 \\
>40,001 \leq 80 \\
>80,001\end{array}$ & $\begin{array}{c}\leq 5 \\
>5,001 \leq 10 \\
>10,001\end{array}$ \\
\hline Índice de humedad y temperatura & $\begin{array}{c}\leq 65 \\
>65,001 \leq 70 \\
>70,001\end{array}$ & $\begin{array}{c}\leq 56 \\
>56,001 \leq 60 \\
>60,001\end{array}$ \\
\hline Edad del animal (meses) & $\begin{array}{c}\leq 45 \\
>45,001 \leq 70 \\
>70,001 \leq 95 \\
>95,001 \leq 120 \\
>120,001\end{array}$ & \\
\hline
\end{tabular}

\section{Análisis de la información}

La información para cada sistema se organizó de forma independiente, en un diseño irrestricto al azar, y se analizó por medio del comando PROC GLM de SAS Institute Inc. (2003). De esta manera, se definió el efecto de las variables independientes sobre la producción de leche diaria (variable dependiente). Como variables independientes se consideraron el mes, el año, el número de lactancia, los días de lactancia, la precipitación diaria, el valor de humedad relativa media, la radiación solar y el índice de humedad. La comparación entre medias de los efectos principales se realizó por medio de la prueba de Duncan (SAS Institute Inc., 2003). En el caso de la edad de los animales, este análisis se realizó con la información obtenida en el sistema de producción ubicado a 600 msnm, donde se definió la edad en semanas y su impacto en la producción de leche en este piso altitudinal. 


\section{Resultados}

\section{Condiciones ambientales registradas en los sistemas de producción durante el periodo de investigación}

Los promedios de temperatura para los sistemas a 660 y $1800 \mathrm{msnm}$ fueron de $22,20{ }^{\circ} \mathrm{C}$ y de $14,99{ }^{\circ} \mathrm{C}$, respectivamente (Cuadro 5). Esta diferencia de $7,21^{\circ} \mathrm{C}$ entre los dos sistemas analizados indica, en esta investigación, que, por cada $158,11 \mathrm{msnm}$ en el incremento de la altura, la temperatura disminuyó en promedio $1^{\circ} \mathrm{C}$. Se observó que la máxima temperatura promedio registrada en el sistema de producción a $660 \mathrm{msnm}$ correspondió al año 2014, con un valor de $23,07^{\circ} \mathrm{C}$, y la mínima en el año 2008 con $21,93^{\circ} \mathrm{C}$. A partir del 2012 y hasta el 2014 , se observó un ascenso anual de temperatura, de 1,18 \% para el período 2012-2013, y de 3,59\% entre los años 2012-2013. En el sistema a $1800 \mathrm{msnm}$, se registró la temperatura promedio más baja en el año 2011, y la máxima en el 2012, posterior a este año, entre 2012 y 2013 , se dio una baja anual en la temperatura de $0,15^{\circ} \mathrm{C}$, de $0,22{ }^{\circ} \mathrm{C}$ entre el 2013 y 2014 , y de $0,04{ }^{\circ} \mathrm{C}$ entre 2014 y 2015 , para una disminución acumulativa de $0,41^{\circ} \mathrm{C}$ entre los años 2012 y 2015. En el caso de la variación mensual de la temperatura, se determinó que para ambos sistemas el mes de mayor temperatura fue junio y el de menor diciembre (Cuadro 5). Mes, en el cual se registró un promedio de temperatura mensual de $22,23^{\circ} \mathrm{C}$ para el sistema a $660 \mathrm{msnm}$, y de $15,12^{\circ} \mathrm{C}$, para el sistema a $1800 \mathrm{msnm}$.

Cuadro 5. Condiciones ambientales e índice de humedad-temperatura (ITH) para los sistemas de producción láctea de hatos jersey, ubicados a 660 y 1800 msnm durante los años investigados. Cartago, Costa Rica. 2016.

Table 5. Environmental conditions and temperature-humidity Index (THI) for milk production systems of jersey herds located at 660 and 1800 m.a.s.l. during the years investigated. Cartago, Costa Rica. 2016.

\begin{tabular}{|c|c|c|c|c|c|c|c|c|c|c|}
\hline \multirow[t]{3}{*}{ Año } & \multicolumn{2}{|c|}{$\begin{array}{c}\text { Temperatura } \\
\left({ }^{\circ} \mathbf{C}\right)^{*}\end{array}$} & \multicolumn{2}{|c|}{$\begin{array}{l}\text { Humedad relativa } \\
\qquad(\%)^{*}\end{array}$} & \multicolumn{2}{|c|}{$\begin{array}{l}\text { Precipitación* } \\
\left(\mathbf{m m} \text { día }^{-1}\right)\end{array}$} & \multicolumn{2}{|c|}{$\begin{array}{l}\text { Radiación } \\
\left(\mathbf{M J ~ m}^{-2}\right)^{*}\end{array}$} & \multicolumn{2}{|c|}{ IHT* } \\
\hline & 660 & 1800 & 660 & 1800 & 660 & 1800 & 660 & 1800 & 660 & 1800 \\
\hline & \multicolumn{2}{|c|}{ msnm } & \multicolumn{2}{|c|}{ msnm } & \multicolumn{2}{|c|}{ msnm } & \multicolumn{2}{|c|}{ msnm } & \multicolumn{2}{|c|}{ msnm } \\
\hline 2003 & $22,22^{\text {cbd }}$ & - & $90,83^{\mathrm{d}}$ & - & $7,70^{\mathrm{a}}$ & - & $14,48^{\mathrm{f}}$ & - & $71,48^{\mathrm{c}}$ & - \\
\hline 2004 & $22,05^{\text {fe }}$ & - & $89,53^{\mathrm{gf}}$ & - & $8,18^{\mathrm{a}}$ & - & $15,31^{\mathrm{de}}$ & - & $71,09^{\mathrm{fe}}$ & - \\
\hline 2005 & $22,24^{\mathrm{cb}}$ & - & $89,33^{\mathrm{g}}$ & - & $7,92^{\mathrm{a}}$ & - & $14,72^{\text {ef }}$ & - & $71,39^{\mathrm{dc}}$ & - \\
\hline 2006 & $22,12^{\mathrm{ced}}$ & - & $87,11^{\mathrm{i}}$ & - & $8,07^{\mathrm{a}}$ & - & $16,63^{\text {ba }}$ & - & $71,02^{\mathrm{fe}}$ & - \\
\hline 2007 & $22,10^{\text {ed }}$ & - & $90,05^{\mathrm{ef}}$ & - & $8,26^{\mathrm{a}}$ & - & $14,96^{\text {ef }}$ & - & $71,22^{\mathrm{de}}$ & - \\
\hline 2008 & $21,93^{\mathrm{fe}}$ & - & $90,39^{\text {ed }}$ & - & $8,70^{\mathrm{a}}$ & - & $14,97^{\text {ef }}$ & - & $70,97^{f}$ & - \\
\hline 2009 & $21,97^{\mathrm{fe}}$ & - & $92,08^{\mathrm{c}}$ & - & $7,63^{\mathrm{a}}$ & - & $16,21^{\mathrm{bc}}$ & - & $71,15^{\text {fe }}$ & - \\
\hline 2010 & $22,25^{\mathrm{cb}}$ & - & $90,89^{d}$ & - & $8,84^{\mathrm{a}}$ & - & $15,74^{\mathrm{dc}}$ & - & $71,56^{\mathrm{c}}$ & - \\
\hline 2011 & $22,10^{\text {ed }}$ & $14,16^{\mathrm{d}}$ & $93,05^{\mathrm{b}}$ & $88,59^{\mathrm{a}}$ & $6,97^{\mathrm{ab}}$ & $3,03^{\mathrm{a}}$ & $17,06^{\mathrm{a}}$ & $11,09^{\mathrm{e}}$ & $71,45^{\mathrm{c}}$ & $57,80^{\mathrm{d}}$ \\
\hline 2012 & $22,01^{\mathrm{fe}}$ & $15,43^{\mathrm{a}}$ & $93,74^{\mathrm{a}}$ & $79,54^{\mathrm{c}}$ & $9,51^{\mathrm{a}}$ & $3,11^{\mathrm{a}}$ & $16,82^{\text {ba }}$ & $14,97^{\mathrm{d}}$ & $71,36^{\mathrm{dc}}$ & $59,88^{\mathrm{a}}$ \\
\hline 2013 & $22,27^{\mathrm{b}}$ & $15,28^{\mathrm{b}}$ & $93,69^{\mathrm{a}}$ & $77,54^{\mathrm{d}}$ & $4,73^{\mathrm{b}}$ & $1,88^{\mathrm{b}}$ & $16,89^{\mathrm{ba}}$ & $16,98^{\mathrm{c}}$ & $71,81^{\mathrm{b}}$ & $59,61^{\mathrm{b}}$ \\
\hline 2014 & $23,07^{\mathrm{a}}$ & $15,06^{\mathrm{c}}$ & $87,89^{\mathrm{h}}$ & $79,99^{c}$ & $7,49^{\mathrm{a}}$ & $0,96^{\mathrm{c}}$ & $15,31^{\mathrm{de}}$ & $37,02^{\mathrm{a}}$ & $72,73^{\mathrm{a}}$ & $59,30^{c}$ \\
\hline 2015 & - & $15,02^{\mathrm{c}}$ & - & $84,44^{\mathrm{b}}$ & - & $0,25^{\mathrm{d}}$ & - & $20,37^{\mathrm{b}}$ & - & $59,27^{\mathrm{c}}$ \\
\hline Promedio & 22,22 & 14,99 & 90,72 & 81,42 & 7,84 & 2,25 & 15,76 & 20,02 & 71,44 & 59,15 \\
\hline
\end{tabular}

* a, b, c, d, e, f, g, h, i medias con letras diferentes dentro de una misma columna difieren entre sí, según la prueba de Duncan (p $\leq 0,05$ ) / means with different letters within the same column differ from other, according Duncan’s test ( $\mathrm{p} \leq 0.05)$.

La humedad relativa (HR) promedio obtenida fue de $90,72 \%$ a $660 \mathrm{msnm}$ y de $82,02 \%$ a $1800 \mathrm{msnm}$, con una diferencia de 8,7 puntos porcentuales. En el sistema a $660 \mathrm{msnm}$, el año que registró el valor mínimo fue el 2006 
con $87 \%$ y el valor máximo fue de $93,74 \%$ en el 2012. La HR presentó diferencias significativas entre años, $\sin$ un patrón claro de aumento o descenso durante el periodo en estudio. El sistema a $1800 \mathrm{msnm}$ presentó un valor mínimo y máximo de HR de 77,54 \% y 84,44 \% en los años 2013 y 2015, respectivamente. En el mes de julio la HR alcanzó su valor máximo en ambos sistemas y presentó una variación en cuanto al mes con el menor valor, en marzo para el sistema a 660 msnm y febrero para la ubicación a 1800 msnm (Cuadro 6).

Cuadro 6. Condiciones ambientales e índice de humedad-temperatura (ITH) para los sistemas de producción láctea de hatos jersey, ubicados a 660 y 1800 msnm durante cada mes.Cartago, Costa Rica. 2016.

Table 6. Environmental conditions and temperature-humidity index (THI) for milk production systems of jersey herd located at 660 and 1800 m.a.s.l. during each month. Cartago, Costa Rica. 2016.

\begin{tabular}{|c|c|c|c|c|c|c|c|c|c|c|}
\hline \multirow[t]{3}{*}{ Mes } & \multicolumn{2}{|c|}{ Temperatura $\left({ }^{\circ} \mathbf{C}\right)^{*}$} & \multicolumn{2}{|c|}{$\begin{array}{c}\text { Humedad relativa } \\
(\%)^{*}\end{array}$} & \multicolumn{2}{|c|}{$\begin{array}{c}\text { Precipitación (mm } \\
\left.\text { día }^{-1}\right)\end{array}$} & \multicolumn{2}{|c|}{ Radiación $\left(\mathrm{MJm}^{-2}\right)^{*}$} & \multicolumn{2}{|c|}{ IHT* } \\
\hline & 660 & 1800 & 660 & 1800 & 660 & 1800 & 660 & 1800 & 660 & 1800 \\
\hline & msnm & msnm & msnm & msnm & msnm & & & & & \\
\hline Enero & $20,78^{j}$ & $14,32^{\mathrm{g}}$ & $90,90^{\mathrm{dc}}$ & $73,70^{\mathrm{e}}$ & $8,48^{\mathrm{b}}$ & $0,23^{\mathrm{h}}$ & $14,39^{\mathrm{e}}$ & $25,41^{\mathrm{c}}$ & $69,01^{\mathrm{h}}$ & $58,09^{\mathrm{h}}$ \\
\hline Febrero & $20,94^{\mathrm{i}}$ & $14,97^{\mathrm{d}}$ & $89,56^{\mathrm{f}}$ & $69,50^{f}$ & $6,52^{c}$ & $0,00^{\mathrm{h}}$ & $16,10^{\mathrm{c}}$ & $29,39^{\mathrm{b}}$ & $69,13^{\mathrm{h}}$ & $59,10^{\mathrm{fe}}$ \\
\hline Marzo & $21,75^{\mathrm{g}}$ & $15,50^{\mathrm{b}}$ & $88,57^{\mathrm{g}}$ & $77,22^{\mathrm{d}}$ & $4,00^{\mathrm{d}}$ & $0,07^{\mathrm{h}}$ & $17,83^{\mathrm{a}}$ & $31,83^{\mathrm{a}}$ & $70,49^{f}$ & $59,89^{4 c}$ \\
\hline Abril & $22,61^{\mathrm{e}}$ & $15,57^{\mathrm{b}}$ & $88,76^{\mathrm{g}}$ & $86,12^{\mathrm{a}}$ & $3,89^{\mathrm{d}}$ & $0,74^{\mathrm{g}}$ & $17,77^{\mathrm{a}}$ & $22,86^{\mathrm{d}}$ & $71,98^{\mathrm{d}}$ & $60,17^{\mathrm{b}}$ \\
\hline Mayo & $22,94^{\mathrm{b}}$ & $15,57^{\mathrm{b}}$ & $90,50^{\text {de }}$ & $82,60^{\mathrm{b}}$ & $8,45^{\mathrm{b}}$ & $1,13^{\mathrm{f}}$ & $17,31^{\mathrm{a}}$ & $23,19^{d}$ & $72,70^{\mathrm{b}}$ & $60,15^{\mathrm{b}}$ \\
\hline Junio & $23,09^{\mathrm{a}}$ & $16,36^{\mathrm{a}}$ & $91,53^{b}$ & $80,57^{\mathrm{c}}$ & $7,63^{\mathrm{cb}}$ & $4,46^{\mathrm{b}}$ & $16,78^{\mathrm{b}}$ & $19,44^{\mathrm{f}}$ & $73,03^{\mathrm{a}}$ & $61,35^{\mathrm{a}}$ \\
\hline Julio & $22,70^{\mathrm{d}}$ & $14,44^{\mathrm{f}}$ & $92,19^{\mathrm{a}}$ & $86,50^{\mathrm{a}}$ & $12,65^{\mathrm{a}}$ & $2,96^{\mathrm{d}}$ & $14,82^{\text {ed }}$ & $15,62^{\mathrm{i}}$ & $72,42^{\mathrm{c}}$ & $58,35^{\mathrm{g}}$ \\
\hline Agosto & $22,95^{\mathrm{b}}$ & $15,09^{\mathrm{c}}$ & $91,05^{\mathrm{c}}$ & $80,97^{c}$ & $7,40^{\mathrm{cb}}$ & $2,74^{\mathrm{d}}$ & $17,47^{\mathrm{a}}$ & $17,89^{\mathrm{h}}$ & $72,75^{\mathrm{b}}$ & $59,35^{\mathrm{d}}$ \\
\hline Septiembre & $22,86^{\mathrm{cb}}$ & $15,50^{\mathrm{b}}$ & $90,32^{\mathrm{e}}$ & $82,17^{b}$ & $6,86^{\mathrm{cb}}$ & $0,86^{\mathrm{gf}}$ & $15,31^{\mathrm{d}}$ & $20,46^{\mathrm{e}}$ & $72,55^{\mathrm{c}}$ & $60,05^{\mathrm{b}}$ \\
\hline Octubre & $22,82^{\mathrm{c}}$ & $14,86^{\mathrm{e}}$ & $90,55^{\text {dce }}$ & $85,82^{\mathrm{a}}$ & $7,33^{\mathrm{cb}}$ & $5,31^{\mathrm{a}}$ & $16,59^{\mathrm{b}}$ & $11,14^{\mathrm{j}}$ & $72,48^{\mathrm{c}}$ & $58,99^{f}$ \\
\hline Noviembre & $21,88^{\mathrm{f}}$ & $15,02^{\mathrm{dc}}$ & $92,11^{\mathrm{a}}$ & $82,51^{\mathrm{b}}$ & $11,13^{\mathrm{a}}$ & $1,54^{\mathrm{e}}$ & $13,34^{\mathrm{f}}$ & $15,79^{i}$ & $70,99^{\mathrm{e}}$ & $59,19^{e}$ \\
\hline Diciembre & $21,49^{\mathrm{h}}$ & $14,17^{\mathrm{h}}$ & $90,71^{\text {dce }}$ & $82,18^{\mathrm{b}}$ & $7,82^{\mathrm{cb}}$ & $3,31^{\mathrm{c}}$ & $13,63^{\mathrm{f}}$ & $18,54^{\mathrm{g}}$ & $70,24^{\mathrm{g}}$ & $57,79^{i}$ \\
\hline Promedio & 22,23 & 15,12 & 90,57 & 80,83 & 7,68 & 1,95 & 15,95 & 20,97 & 71,49 & 59,38 \\
\hline
\end{tabular}

*a, b, c, d, e, f, g, h, i, j medias con letras diferentes dentro de una misma columna difieren entre sí, según la prueba de Duncan (p $\leq 0,05)$ / means with different letters within the same column differ from other, according Duncan's test ( $\mathrm{p} \leq 0.05)$.

En el caso de la precipitación, el valor promedio obtenido en los sistemas de producción analizados fue de 7,83 $\mathrm{mm}$ día $^{-1}$ a $660 \mathrm{msnm}$ y de $1,85 \mathrm{~mm} \mathrm{día}^{-1}$ a $1800 \mathrm{msnm}$, con una diferencia de 5,98 $\mathrm{mm}$ día $^{-1}$ entre sí. En el sistema a $660 \mathrm{msnm}$ se registró un valor máximo de $9,51 \mathrm{~mm}_{\text {día }}^{-1}$ durante el 2012 y un mínimo de 4,73 mm día ${ }^{-1}$ durante el 2013, con una variación máxima de 4,78 $\mathrm{mm} \mathrm{día}^{-1}$ en el período de evaluación. A $1800 \mathrm{msnm}$, el máximo de precipitación fue de $3,11 \mathrm{~mm} \mathrm{día}^{-1}$ y el mínimo de $0,25 \mathrm{~mm}_{\text {día }}^{-1}$; en este sistema, se dio una disminución anual de la precipitación de $1,23 \mathrm{~mm}_{\text {día }}^{-1}$ entre los años 2012 y 2013, de $0,92 \mathrm{~mm} \mathrm{día}^{-1}$ entre el 2013 y 2014, y de 0,71 mm día ${ }^{-1}$ entre el 2014 y el 2015. Lo que totaliza una disminución en la precipitación de 2,86 mm día ${ }^{-1}$ o su equivalente a 85,8 $\mathrm{mm} \mathrm{mes}^{-1}$. El comportamiento mensual de la precipitación se registra en el Cuadro 6, siendo el mes de julio el de mayor volumen y abril el de menor, para el sistema de menor altitud; mientras que a $1800 \mathrm{msnm}$, octubre fue el mes de mayor precipitación y febrero donde no se contabilizóprecipitación.

La radiación presentó valores promedio de $15,76 \mathrm{MJ} \mathrm{m}^{-2}$ y de 20,09 $\mathrm{MJ} \mathrm{m}^{-2}$ para los sistemas de producción ubicados a 660 y $1800 \mathrm{msnm}$, respectivamente. A $660 \mathrm{msnm}$ se presentó un máximo de radiación de 17,06 MJ m² 
en el 2011 y un mínimo de 14,48 MJ m² durante el 2003 (p<0,05). En cambio, a $1800 \mathrm{msnm}$ se registró un aumento en los valores de la radiación desde el 2011 hasta el 2014, con un valor mínimo de 11,09 MJ m² y un máximo de 37,02 $\mathrm{MJ} \mathrm{m}^{-2}$. La fluctuación mensual de la radiación reveló que marzo fue el período del año con la mayor radiación en ambos sistemas, y noviembre y octubre fueron los meses con menor radiación registrados para los sistemas a 660 y $1800 \mathrm{msnm}$, respectivamente (Cuadro 6). En la altitud de $660 \mathrm{~m}$ se registró una mayor temperatura y humedad relativa en relación con la altitud de $1800 \mathrm{~m}$, lo cual concuerda con el IHT promedio determinado para cada uno, de 71,44 y 59,18, respectivamente (Cuadro 5).

Factores temporales que se relacionan con la producción de leche de los semovientes en cada piso altitudinal

El promedio de producción de los años evaluados del sistema de producción láctea ubicado a $660 \mathrm{msnm}$ fue de 15,23 kg leche día $^{-1}$ y de 19,95 kg leche lía $^{-1}$ para los animales desarrollados a una altitud de $1800 \mathrm{~m}$ (Cuadro 7). Al comparar los sistemas productivos durante años similares (2011-2012-2013-2014), se obtuvo que la producción promedio de leche por día por animal fue de $16,50 \pm 0,34 \mathrm{~kg}$ y de $20,20 \pm 0,89 \mathrm{~kg}$ a 660 y $1800 \mathrm{msnm}$, respectivamente. La producción láctea promedio de las vacas estuvo afectada significativamente de un mes a otro durante el año $(\mathrm{p}<0,05)$. El promedio de producción mensual fue de $15,41 \mathrm{~kg}^{\text {leche-1 } \mathrm{dí}^{-1} \mathrm{y} \text { de } 20,4 \mathrm{~kg} \text { leche }}$ ${ }^{1}$ día $^{-1}$ para los sistemas a 660 y $1800 \mathrm{msnm}$, respectivamente. En la Figura 1 se muestran las dos tendencias en la producción láctea en cada sistema durante el año. A la altitud de a $660 \mathrm{~m}$ se observó que, en los primeros cinco meses del año (enero-mayo), hubo las mayores producciones promedio, donde la máxima se alcanzó en los meses de abril y mayo $\left(16,26 \mathrm{~kg}_{\text {leche }}^{-1} \mathrm{día}^{-1}\right)$; en cambio, el sistema localizado a $1800 \mathrm{msnm}$ registró un aumento en el

Cuadro 7. Producción láctea de hatos jersey, en cada piso altitudinal, según el año de evaluación. Cartago, Costa Rica. 2016.

Table 7. Dairy milk production of jersey herd in each altitudinal level according to the year of evaluation. Cartago, Costa Rica. 2016.

\begin{tabular}{|c|c|c|}
\hline \multirow[t]{2}{*}{ Año } & \multicolumn{2}{|c|}{ Producción de leche* (kg día ${ }^{-1}$ animal $\left.^{-1}\right)$} \\
\hline & $660 \mathrm{msnm}$ & $1800 \mathrm{msnm}$ \\
\hline 2003 & $14,55^{\mathrm{ed}}$ & - \\
\hline 2004 & $14,00^{\mathrm{fg}}$ & - \\
\hline 2005 & $14,12^{\text {efg }}$ & - \\
\hline 2006 & $13,63^{\mathrm{g}}$ & - \\
\hline 2007 & $14,17^{\mathrm{ef}}$ & - \\
\hline 2008 & $15,31^{\mathrm{c}}$ & - \\
\hline 2009 & $14,73^{\mathrm{d}}$ & - \\
\hline 2010 & $16,20^{\mathrm{b}}$ & - \\
\hline 2011 & $16,44^{\mathrm{b}}$ & $20,39^{b}$ \\
\hline 2012 & $16,43^{\mathrm{b}}$ & $20,56^{\mathrm{b}}$ \\
\hline 2013 & $16,95^{\mathrm{a}}$ & $20,94^{\mathrm{a}}$ \\
\hline 2014 & $16,13^{\mathrm{b}}$ & $18,90^{\mathrm{c}}$ \\
\hline 2015 & - & $18,93^{\mathrm{c}}$ \\
\hline Promedio & $15,23 \pm 1,16$ & $19,95 \pm 0,96$ \\
\hline
\end{tabular}

*a, b, c, d, e, f, g medias con letras diferentes dentro de una misma columna difieren entre sí, según la prueba de Duncan ( $\mathrm{p} \leq 0,05$ ) / means with different letters within the same column differ from other, according Duncan's test ( $\mathrm{p} \leq 0.05)$. 


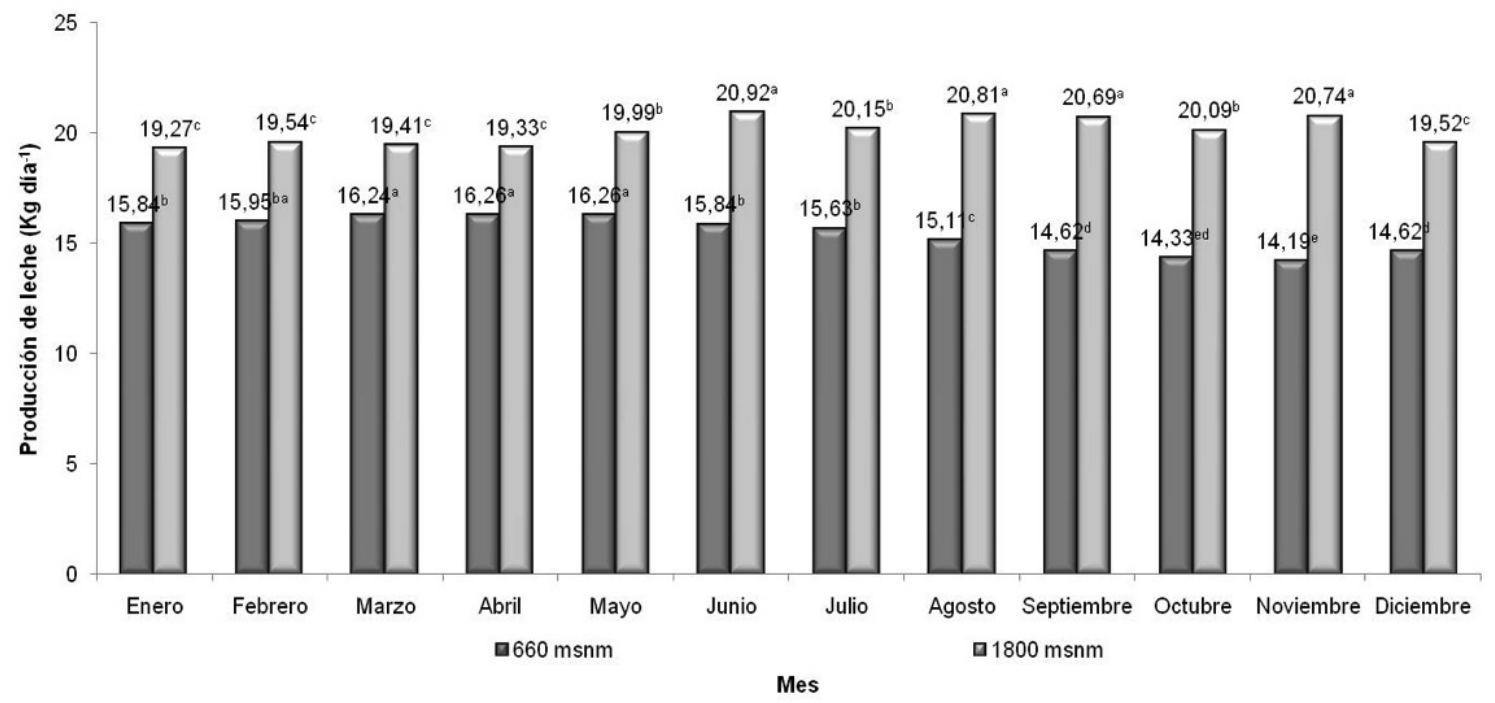

Figura 1. Comportamiento de la producción láctea a través del año en los sistemas de producción láctea de hatos jersey, ubicados a 660 y 1800 msnm. Cartago, Costa Rica. 2016.

Figure 1. Behavior of dairy milk production throughout the year in milk production systems of jersey herd at 660 and 1800 m.a.s.1. Cartago, Costa Rica. 2016.

volumen de producción en los últimos seis meses del año (junio-diciembre), período en el que se alcanzó el pico de producción en el mes de agosto, con un valor de 20,81 kg leche día $^{-1}$.

\section{Factores intrínsecos del animal relacionados con la producción diaria de leche}

El efecto individual de cada vaca sobre la producción de leche presentó alta significancia $(\mathrm{p}<0,001)$. De igual manera, el número de lactancia que registró cada animal durante la investigación (Cuadro 8). Para el sistema de producción láctea ubicado a $660 \mathrm{msnm}$, el valor máximo se alcanzó en la tercera lactancia con 16,54 kg de leche diarios. En este sistema, las lactancias 3, 4, 5, y 6, no presentaron diferencias significativas, también se obtuvo una similitud en producción $(\mathrm{p}<0,05)$ entre animales de primera y más de siete lactancias. En el sistema situado a 1800 msnm se determinó que la mayor producción láctea estuvo entre la tercera y décima lactancia $(\mathrm{p}<0,05)$. En el Cuadro 9, se observa que aquellos en el sistema a $600 \mathrm{msnm}$ se registró la producción de leche más elevado entre los 45,1 y los 70 meses de edad, con valores que superaron en 0,821 día $^{-1}$ a animales con edades menores a los 45 meses y en 0,17 1 día $^{-1}$ a aquellos que superaban los 70,1 meses de edad. En el caso de los animales con edades mayores a los 120,1 meses (diez años), se determinó una reducción en la producción de leche de 3,7 litros por día, al compararse con animales entre los 45,1 y 70 meses (3,7 a 5,8 años).

El comportamiento de la producción láctea durante el período de lactancia en ambos sistemas se describe en el Cuadro 10. En el caso del sistema a $660 \mathrm{msnm}$, se alcanzó un pico de producción de 19,24kg de leche diarios entre los 30 y 45 días postparto. Mientras que, en el sistema a $1800 \mathrm{msnm}$ el pico de producción láctea se registró entre los 15 y los 30 días postparto, con un valor de $23,91 \mathrm{~kg}$ de leche diarios. La persistencia determinada en cada sistema fue de $95,86 \pm 3,49$ y de $98,20 \pm 5,85$ para 660 y $1800 \mathrm{msnm}$, respectivamente. 
Cuadro 8. Producción láctea de animales jersey según el número de lactancia en dos pisos altitudinales. Cartago, Costa Rica. 2016.

Table 8. Milk production of jersey animals according to the number of lactation in two altitudinal levels. Cartago, Costa Rica. 2016.

\begin{tabular}{|c|c|c|}
\hline \multirow[t]{2}{*}{ Lactancia } & \multicolumn{2}{|c|}{ Producción de leche (kg día-1)* } \\
\hline & $660 \mathrm{msnm}$ & $1800 \mathrm{msnm}$ \\
\hline 1 & $14,16^{\mathrm{b}}$ & $17,76^{\mathrm{e}}$ \\
\hline 2 & $15,98^{\text {a }}$ & $20,05^{\text {bdc }}$ \\
\hline 3 & $16,54^{\mathrm{a}}$ & $20,49^{\text {bac }}$ \\
\hline 4 & $16,40^{\text {a }}$ & $21,60^{\text {a }}$ \\
\hline 5 & $16,00^{\text {a }}$ & $21,48^{\text {bac }}$ \\
\hline 6 & $15,70^{\text {a }}$ & $21,41^{\text {bac }}$ \\
\hline 7 & $12,40^{\mathrm{c}}$ & $21,20^{\text {bac }}$ \\
\hline 8 & $13,90^{\mathrm{b}}$ & $21,88^{\text {a }}$ \\
\hline 9 & $12,14^{\mathrm{c}}$ & $20,99^{\text {bac }}$ \\
\hline 10 & $10,78^{\mathrm{d}}$ & $19,88^{\text {bac }}$ \\
\hline 11 & & $18,83^{\text {ed }}$ \\
\hline Promedio & $14,40 \pm 2,05$ & $20,51 \pm 1,22$ \\
\hline
\end{tabular}

*a, b, c, d, e, medias con letras diferentes dentro de una misma columna difieren entre sí, según la prueba de Duncan ( $\leq 0.05)$ / means with different letters within the same column differ from other, according Duncan's test ( $\mathrm{p} \leq 0.05)$.

Cuadro 9. Relación de la edad del animal y la producción láctea de animales jersey ubicados a 660 msnm. Turrialba, Cartago, Costa Rica. 2016.

Table 9. Relationship of the animal age and the dairy production of jersey animals located at 660 m.a.s.l. Turrialba, Cartago, Costa Rica. 2016

\begin{tabular}{|c|c|}
\hline \multirow[t]{2}{*}{ Rangos de edad del animal (meses) } & Producción de leche (kg día $\left.{ }^{-1}\right)^{*}$ \\
\hline & $660 \mathrm{msnm}$ \\
\hline$\leq 45$ & $15,12^{b}$ \\
\hline$>45,1 \leq 70$ & $15,94^{\text {a }}$ \\
\hline$>70,1 \leq 95$ & $15,77^{\text {a }}$ \\
\hline$>95,1 \leq 120$ & $14,95^{b}$ \\
\hline$>120,1$ & $12,24^{\mathrm{c}}$ \\
\hline
\end{tabular}

*a, b, c medias con letras diferentes dentro de una misma columna difieren entre sí, según la prueba de Duncan $(\mathrm{p} \leq 0,05)$ / means with different letters with in the same column differ from other, according Duncan's test ( $\mathrm{p} \leq 0.05)$.

\section{Factores ambientales relacionados con la producción diaria de leche de animales jersey}

Para el hato ubicado a $660 \mathrm{msnm}$, los valores de producción láctea no presentaron diferencias significativas ( $p>0,05$ ) según el rango de temperatura analizado (Figura 2). Para la ubicación de $1800 \mathrm{msnm}$, existió una diferencia significativa en la producción láctea de los animales a temperaturas menores a $14{ }^{\circ} \mathrm{C}$, la cual tendió a disminuir, 
Cuadro 10. Producción láctea de animales jersey en dos pisos altitudinales, según los días de lactancia. Cartago, Costa Rica. 2016.

Table 10. Milk production of jersey animals on two altitudinal level according to the days of lactation. Cartago, Costa Rica. 2016.

\begin{tabular}{|c|c|c|}
\hline \multirow[t]{2}{*}{ Rangos de días de lactancia * } & \multicolumn{2}{|c|}{ Producción de leche $\left(\mathrm{kg} \mathrm{día}^{-1}\right)^{* *}$} \\
\hline & $660 \mathrm{msnm}$ & $1800 \mathrm{msnm}$ \\
\hline$\leq 15$ & 19,24 fe & $21,71^{\mathrm{c}}$ \\
\hline$>15,01 \leq 30$ & 18,87 ba & $23,91^{\text {a }}$ \\
\hline$>30,01 \leq 45$ & $19,22^{a}$ & $23,07^{\text {a }}$ \\
\hline$>45,01 \leq 60$ & $19,16^{\mathrm{a}}$ & $23,48^{\text {a }}$ \\
\hline$>60,01 \leq 75$ & 18,65 ba & 23,34 ba \\
\hline$>75,01 \leq 90$ & $17,92^{\mathrm{c}}$ & 22,68 ba \\
\hline$>90,01 \leq 105$ & $17,54^{\mathrm{c}}$ & $21,65^{\mathrm{c}}$ \\
\hline$>105,01 \leq 130$ & $16,85^{\mathrm{d}}$ & $20,80^{\mathrm{d}}$ \\
\hline$>130,01 \leq 145$ & $16,31^{\mathrm{e}}$ & 20,08 ed \\
\hline$>145,01 \leq 150$ & 15,95 fe & 17,70 ed \\
\hline$>150,01 \leq 175$ & $15,60 \mathrm{fe}$ & $18,92^{\mathrm{f}}$ \\
\hline$>175,01 \leq 200$ & $14,83^{\mathrm{g}}$ & $18,22^{\mathrm{f}}$ \\
\hline$>200,01 \leq 225$ & $14,31^{\mathrm{h}}$ & $17,42^{\mathrm{g}}$ \\
\hline$>225,01 \leq 250$ & $13,80^{\mathrm{i}}$ & $16,81^{\mathrm{g}}$ \\
\hline$>250,01 \leq 300$ & $13,04^{\mathrm{j}}$ & $15,99^{\mathrm{h}}$ \\
\hline$>300,01 \leq 350$ & $12,54^{\mathrm{k}}$ & $15,24^{\mathrm{i}}$ \\
\hline$>350,01 \leq 400$ & $12,40^{\mathrm{k}}$ & $15,09^{\mathrm{i}}$ \\
\hline$>400,01 \leq 500$ & $11,44^{1}$ & $17,16^{\mathrm{g}}$ \\
\hline$>500,001$ & $9,67^{\mathrm{m}}$ & \\
\hline
\end{tabular}

*( $\leq$ ) Menor o igual/ Less or equal; $(>\mathrm{X} \leq \mathrm{Y})$ Mayor a X pero menor o igual a Y/ Greater than $\mathrm{X}$ but less than or equal to Y, y (>) Mayor a/ Greater than.

$* * a, b, c, d, e, f, g, h, i, j, k$ medias con letras diferentes dentro de una misma columna difieren entre sí, según la prueba de Duncan (p $\leq 0.05$ ) / means with different letters within the same column differ from other, according Duncan’s test ( $\mathrm{p} \leq 0.05)$.
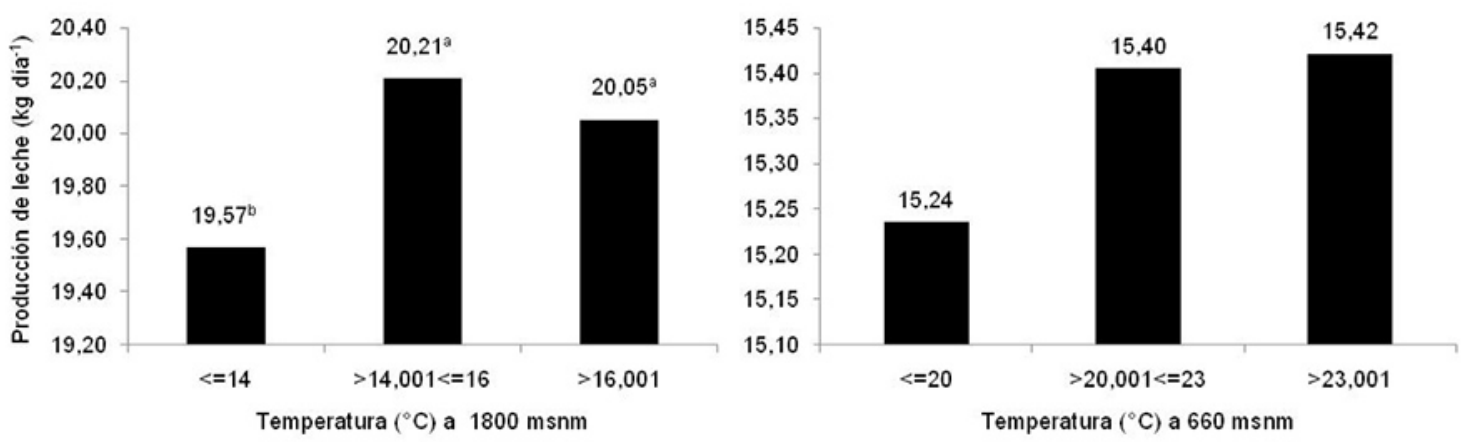

Figura 2. Relación entre la temperatura registrada y la producción láctea de dos hatos de animales jersey, uno ubicado a 660 y otro a 1800 msnm. Cartago, Costa Rica. 2016.

a,b Letras diferentes entre columnas difieren entre sí, según la prueba de Duncan $(\mathrm{p} \leq 0,05)$.

Figure 2. Relationship between the recorded temperature and the milk production of two Jersey herds located at 660 and the other at 1800 m.a.s.l. Cartago, Costa Rica. 2016.

$\mathrm{a}, \mathrm{b}$ Different letters between columns differ from other, according Duncan's test $(\mathrm{p} \leq 0.05)$. 
ya que el mayor potencial productivo de las vacas estuvo entre los 14 y $16{ }^{\circ} \mathrm{C}$, con una producción promedio por animal por día de 20,21 $\mathrm{kg}_{\text {leche }} \mathrm{día}^{-1}$. La producción de leche alcanzó su punto máximo cuando los valores de precipitación superaron los $80 \mathrm{~mm} \mathrm{día}^{-1}$ en el sistema a $660 \mathrm{msnm}$ (Figura 3). Existió una diferencia significativa entre la precipitación y la producción láctea para el sistema ubicado a $1800 \mathrm{msnm}$, donde se registró la mayor producción (20,6 kg de leche diarios) cuando se presentaron entre los 5 y $10 \mathrm{~mm} \mathrm{día}^{-1}$. Valores inferiores o iguales a $5 \mathrm{~mm}$ día $^{-1}$ provocaron una disminución de $0,62 \mathrm{~kg}$ de leche diarios respecto del rango situado entre los 5 y $10 \mathrm{~mm}$ día ${ }^{-1}$ (Figura 3). De igual manera, se observó una disminución de 0,48 $\mathrm{kg}$ de leche diarios cuando la precipitación presentó valores superiores a $10 \mathrm{~mm} \mathrm{día}^{-1}$.
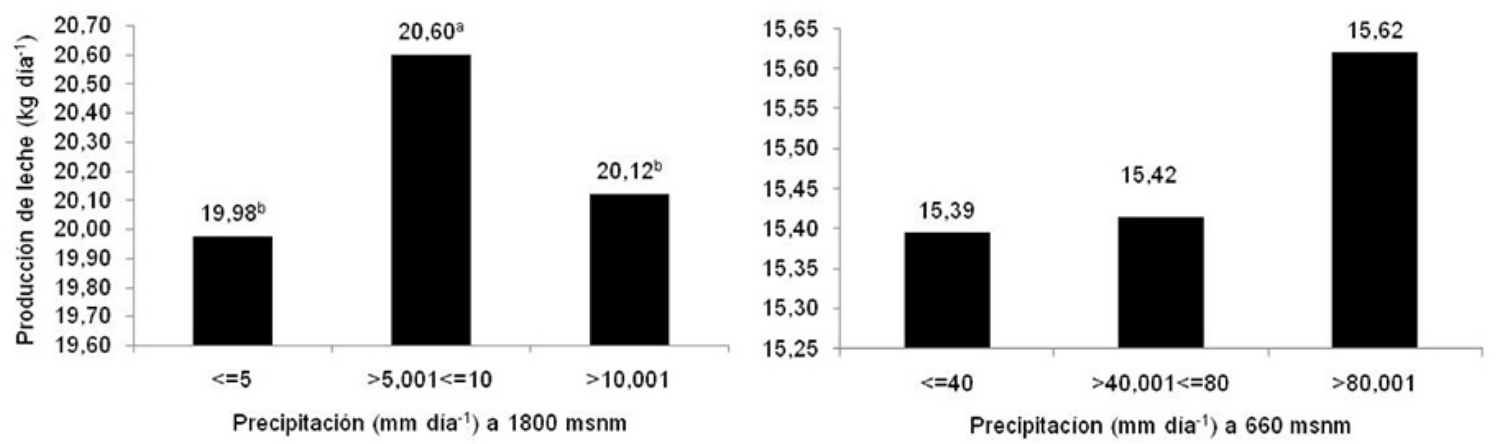

Figura 3. Relación entre la precipitación registrada y la producción láctea de dos hatos de animales jersey, uno ubicado a 660 y otro a 1800 msnm. Cartago, Costa Rica. 2016.

a, b Letras diferentes entre columnas difieren entre sí, según la prueba de Duncan $(\mathrm{p} \leq 0,05)$.

Figure 3. Relationship between the recorded precipitation and the milk production of two jersey herds one located at 660 and the other at 1800 m.a.s.l. Cartago, Costa Rica. 2016.

$\mathrm{a}, \mathrm{b}$ Different letters between columns differ from other, according Duncan's test $(\mathrm{p} \leq 0.05)$.

Con respecto a la evaluación de la radiación en ambos sistemas, esta presentó un efecto diferente según el piso altitudinal, a $660 \mathrm{msnm}$ se registró un aumento de $0,60 \mathrm{~kg}$ de leche diarios en rangos de radiación superiores a 20 MJ m-2, en cambio, a 1800 msnm el máximo valor de producción se alcanzó cuando la radiación fue menor o igual a $10 \mathrm{MJ} \mathrm{m}^{-2}$ (Figura 4).

La producción de leche a $660 \mathrm{msnm}$ alcanzó su máximo valor cuando la HR fue menor o igual a $70 \%$ y disminuyó cuando la HR superó el $80 \%$ (Figura 5). Cuando la HR pasó de 70 a $90 \%$, los animales dejaron de producir 5,07 l de leche, o redujeron la productividad en $169 \mathrm{ml}$ de leche por día por cada aumento porcentual en el contenido de humedad del aire. La producción de leche a 1800 msnm alcanzó su máxima producción promedio en valores de humedad relativa mayores a $80 \%$ y menores o iguales a $90 \%$ (Figura 5). Se observó que los mínimos valores de producción se presentaron en el rango de humedad relativa menor o igual a $70 \%$ y mayor a $90 \%$. Entre los rangos de 70 y $80 \%$, y del 80 al $90 \%$ de humedad, se registró un aumento en el promedio de producción de $0,15 \mathrm{~kg}$ de leche diarios $(\mathrm{p}<0,05)$; pero cuando la humedad superó el $90 \%$, se registró una disminución de 0,62 $\mathrm{kg}$ de leche diarios.

En el sistema ubicado a $660 \mathrm{msnm}$, conforme aumentó el índice de humedad-temperatura disminuyó el promedio diario de producción láctea en 1,35 1 (Figura 6), mientras que cuando este indicador alcanzó valores menores a 65, la producción láctea fue superior. En el caso del sistema ubicado a $1800 \mathrm{msnm}$, se determinó una 

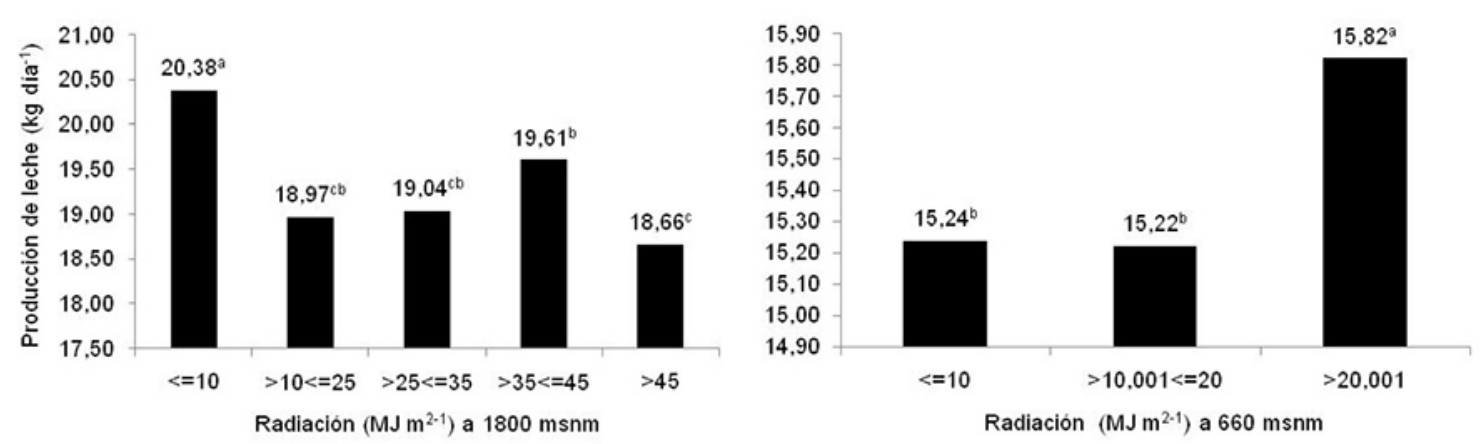

Figura 4. Relación entre la radiación registrada y la producción láctea de dos hatos de animales jersey, uno ubicado a 660 y otro a 1800 msnm. Cartago, Costa Rica. 2016.

a, b, c Letras diferentes entre columnas difieren entre sí, según la prueba de Duncan $(\mathrm{p} \leq 0,05)$.

Figure 4. Relationship between the registered radiation and the milk production of two jersey herds, one located at 660 and the other at 1800 m.a.s.l. Cartago, Costa Rica. 2016.

$\mathrm{a}, \mathrm{b}, \mathrm{c}$ Different letters between columns differ from other, according Duncan's test ( $\mathrm{p} \leq 0.05)$.
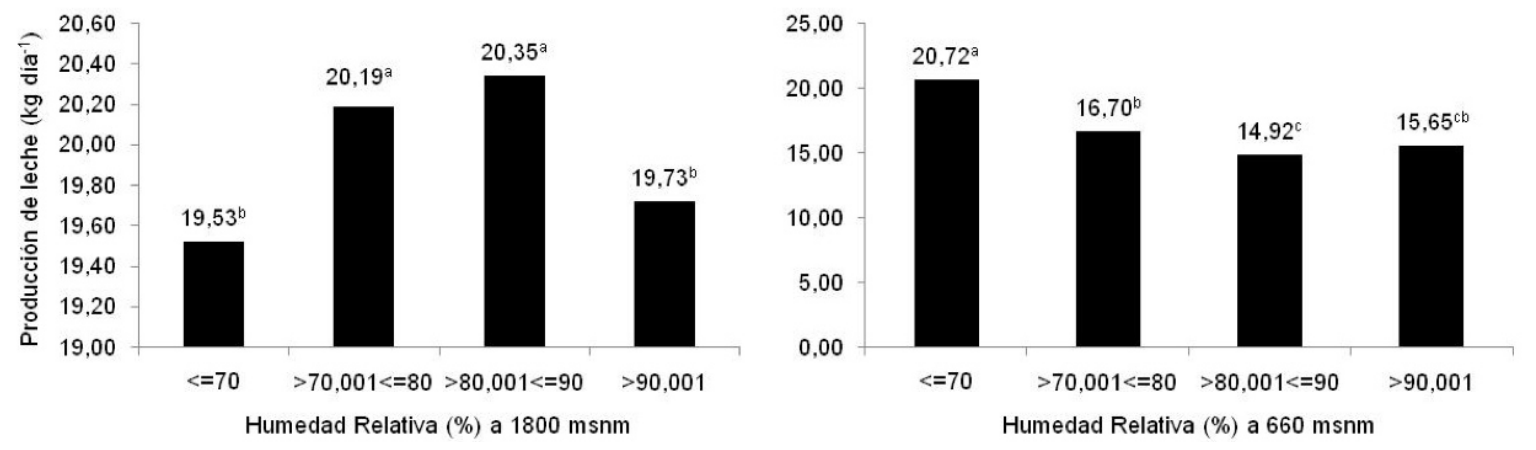

Figura 5. Relación entre la humedad relativa registrada y la producción láctea de dos hatos de animales jersey, uno ubicado a 660 y otro a 1800 msnm. Cartago, Costa Rica. 2016.

a, b, c Letras diferentes entre columnas difieren entre sí, según la prueba de Duncan ( $\mathrm{p} \leq 0,05)$.

Figure 5. Relationship between the recorded relative humidity and the milk production of two jersey herds, one located at 660 and the other at 1800 m.a.s.l. Cartago, Costa Rica. 2016.

$\mathrm{a}, \mathrm{b}, \mathrm{c}$ Different letters between columns differ from other, according Duncan's test $(\mathrm{p} \leq 0.05)$.

tendencia contraria a la encontrada a $660 \mathrm{msnm}$, donde conforme aumentó el índice de humedad-temperatura aumentó el promedio diario de producción láctea. Asimismo, se observó que el valor máximo de producción láctea se alcanzó en índices de humedad-temperatura mayores a 60; mientras que valores menores o iguales a 56 mostraron la menor producción láctea diaria.

\section{Discusión}

En el caso de las variaciones climáticas determinadas en este trabajo, los especialistas en esta área recomiendan el análisis de periodos de diez años como mínimo, debido a que se podría incurrir en el error de evaluar los cambios 

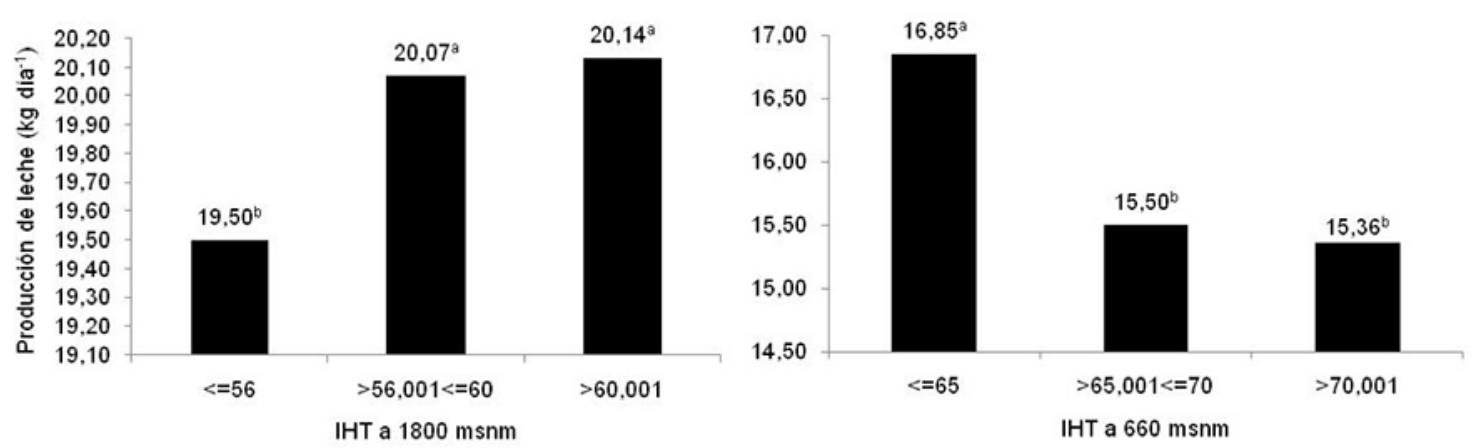

Figura 6. Relación entre índice humedad-temperatura determinado y la producción láctea de dos hatos de animales jersey, uno ubicado a 660 y otro a 1800 msnm. Cartago, Costa Rica. 2016.

a, b Letras diferentes entre columnas difieren entre sí, según la prueba de Duncan $(\mathrm{p} \leq 0,05)$.

Figure 6. Relationship between the determined temperature-humidity index and the milk production of two jersey herds located at 660 andthe other at 1800 m.a.s.l. Cartago, Costa Rica. 2016.

$\mathrm{a}, \mathrm{b}$ Different letters between columns differ from other, according Duncan's test $(\mathrm{p} \leq 0.05)$.

del tiempo y no la tendencia actual del clima (Vargas, 2006). Las variaciones ambientales podrían estar asociadas al crecimiento de la población a nivel global, que aumentan la concentración de gases de efecto invernadero, al cambio del uso del suelo que reducen las áreas de captura y que interactúan con los cambios de clima de forma natural, sumado a las características de Costa Rica, la cual se encuentra entre el mar Caribe y el Océano Pacífico (IMN, 2008). En el caso de la radiación, la diferencia de 4,33 MJ m² encontrada, es explicada por los $1140 \mathrm{msnm}$ de diferencia entre un sistema y otro, lo cual concuerda con lo informado por el Instituto Meteorológico Nacional de Costa Rica (IMN, 2015), quien indicó, que a mayor altitud menor será la atenuación de los rayos del sol por la atmósfera, por lo que la radiación UV será mayor que a nivel del mar.

Estas variaciones provocan que los animales experimenten estrés (Dikmen y Hansen, 2009) y que requieran de una mayor inversión energética para la disipación de calor, lo cual limita su capacidad productiva (Collier et al., 2006), debido a que recurren al desvío de la energía hacia el mantenimiento de la temperatura corporal, a través de la activación de sistemas corporales con fines termorreguladores, a la modificación en el ritmo de secreción hormonal, que modifica los índices metabólicos, y a la reducción en el consumo de alimento, con la consecuente disminución en la ganancia de peso y en la producción de carne o leche (Alcina et al., 2001). En el caso del recurso forrajero, disminuciones en la precipitación podrían afectar el crecimiento del forraje y, en consecuencia reducir la cantidad disponible de esta fuente alimentaria para los animales.

Con base en la producción láctea de animales jersey según el número de lactancia en dos pisos altitudinales (Cuadro 7), se mostró que los semovientes a $1800 \mathrm{msnm}$, presentaron mayor producción y vida productiva, situación que se denotó, al comparar la productividad de animales con más de diez lactancias, con promedios de producción, que superaron en 2,29 1 de leche por día a los animales de tercera lactancia a $660 \mathrm{msnm}$, donde, en lactancias superiores a 250 días, se obtuvieron 572,5 1 de leche de más en cada ciclo productivo. En el caso del sistema a menor altura, permite implementar un sistema de selección de animales, con la meta de obtener la mayor productividad de los animales presentes, debido a que la vida productiva de las vacas no podría superar los diez años, ya que producen menos leche al día y compiten por forraje y alimento balanceado con animales de menor edad y mayor producción. Para el sistema de mayor altura, la constancia en el promedio de producción por animal entre lactancias, podría indicar problemas de manejo propios del sistema a nivel nutricional o una barrera natural que limita el potencial de los animales que pastorean forrajes tropicales, debido a que, según Carvajal et al. (2002), la mayor producción de leche se obtiene entre la tercera y la quinta lactancia, momento en el cual la vaca alcanza su peso adulto. 
La diferencia promedio de 3,7 kg leche $\mathrm{kía}^{-1}$ por animal entre los sistemas productivos (2011 al 2014) se podría explicar por los días de lactancia promedio del hato, el manejo alimenticio, los problemas reproductivos (Lucy, 2001) y por las condiciones ambientales presentes en cada ecosistema (Pérez y Gómez, 2005), las cuales se relacionan con la altitud y con el tipo, la oferta y la calidad nutricional del forraje que se les suministra al ganado.

En el sistema a $660 \mathrm{msnm}$ los animales pastorearon estrella africana, el cual tiene $1,23 \mathrm{Mcal} \mathrm{kg}^{-1} \mathrm{EN}_{\mathrm{L}}$ de $\mathrm{MS}$, $54,1 \%$ de nutrientes digestibles totales y $15,3 \%$ de proteína cruda. En cambio, en el sistema a $1800 \mathrm{msnm}$ se utilizó el pasto kikuyo, el cual contiene 1,30 Mcal kg-1 de MS, 56,7\% de nutrientes digestibles totales y 17,4\% proteína cruda en su perfil nutricional (Sánchez y Soto, 1999). Por tanto, un kilogramo de materia seca de ambos materiales presenta una concentración porcentual a favor del kikuyo de 5,69 \% de ENL, 4,80 \% NDT y 13,73 \% de PC, que aporta más nutrimentos al animal; sin considerar el efecto sobre el consumo voluntario de alimento y el bienestar animal que presenten estas condiciones al generar periodos de estrés en el semoviente (Silanikove, 2000). Otras consideraciones que explican estas diferencias en producción, según el día de lactancia, se podrían asociar a la condición corporal (Stadnik et al., 2017), el número de lactancia y a la edad del animal que presentó cada animal al momento de la recolección de la información (Haworth et al., 2008), sumado al genotipo de los animales y la respuesta a la interacción con el ambiente, este último incluiría el manejo alimenticio y las condiciones ambientales que se registran en estos sistemas (Soydan y Kuran, 2017), como la variación de la temperatura y la humedad relativa.

Según lo determinado en esta investigación (Cuadro 1), el sistema de producción ubicado a $1800 \mathrm{msnm}$ presentó $7,23{ }^{\circ} \mathrm{C}$ menos, $9,3 \%$ más de $\mathrm{HR}$, una menor incidencia de radiación $\left(4,26 \mathrm{MJ} \mathrm{m}^{-2}\right)$ y 12,29 puntos menos en el IHT, diferencias que afectaron la productividad de los animales. En aspectos genéticos, el sistema ubicado a 1800 msnm, como única escogencia en el programa de inseminación que implementan, se utilizó el animal que se encuentra con mejor calificación de tipo y conformación para la raza jersey, según la evaluación internacional.

El efecto del piso altitudinal en el cual se encontraban los semovientes y de la HR afectaron de forma diferente la productividad de los animales, lo cual indica una interacción entre la temperatura y la humedad del aire, y la productividad de los animales. Según Arias et al. (2008) los principales efectos de la HR están asociados a la poca capacidad que presenta el aire cuando presenta altos contenidos de humedad para intercambiar calor por medio de la sudoración y respiración en los semovientes. En cambio, desde una perspectiva del cultivo, como principal fuente de alimento, según Quintero et al. (1995) se ofrece al animal un forraje de mejor calidad, debido a la relación inversa entre el aumento de la humedad relativa y la disminución de la tasa de evaporación y transpiración de las plantas, lo que permite una disminución de la tasa de madurez, por un mejor aprovechamiento del agua del suelo. Caso contrario, cuando la temperatura ambiental es alta y se presenta baja humedad relativa, debido a que se aceleran los procesos de lignificación y conversión de productos de la fotosíntesis en materia estructural del cultivo (Van-Soest, 1994).

En el caso del IHT, los resultados obtenidos concuerdan con la literatura, en donde se establece un tope de 72 de IHT para valorar condiciones de estrés en los semovientes (Polsky y von-Keyserlingk, 2017). Es importante resaltar que este valor se estableció en condiciones de humedad y temperatura en otras latitudes, y que en condiciones tropicales como las que se presentan en Turrialba, Costa Rica $(660 \mathrm{msnm})$, este valor se ajusta a 65 de IHT (7 puntos menos de los que indica la literatura), ya que a partir de este valor se inicia la merma en la productividad de los animales, la cual se podría relacionar a problemas de estrés calórico por parte de los animales, que de acuerdo con Staples y Thatcher (2011), cuando el ITH es alto, la disipación de calor por parte del animal es más difícil, ya que la producción de calor de la vaca aumenta cuando está en un estado metabólico de la lactancia, por un mayor consumo de materia seca.

En el sistema de producción que se encontraba a $1800 \mathrm{msnm}$, el IHT presentó un efecto inverso, debido a que se generó un indicador de estrés por frío, tal como se observó la respuesta de los animales con IHT por debajo de 56, donde se presentaron mermas de producción cercanas a $570 \mathrm{ml}$ de leche por día. Situación que podría indicar que los animales a bajas temperaturas destinan parte de los nutrimentos digeridos para labores de calentamiento, o sufren periodos de bajos consumos de alimento cuando se agrupan en el campo para evitar la pérdida de calor. En 
este caso, se podría indicar que el ganado a temperaturas menores de $14{ }^{\circ} \mathrm{C}$ sufre estrés por frío y que por encima de $16^{\circ} \mathrm{C}$ presenta proceso de estrés por calor, situación que se podría asociar a que los animales no están adaptados a altas temperaturas. Otro aspecto a valorar es que en la zona alta de Cartago, Costa Rica, cuando la temperatura baja a valores cercanos al punto de congelación, se puede dar el fenómeno de escarchado en los pastos. El daño ocurre cuando se forma hielo dentro del tejido de las plantas, lo que rompe las células, y tiene un efecto drástico para la planta entera o puede afectar a una pequeña parte de su tejido, lo cual reduce el rendimiento o deprecia la calidad del material (Snyder et al., 2010).

En estudios de esta índole, es importante considerar los rangos de temperatura, precipitación, radiación y humedad relativa (Cuadro 2 y 3), debido a que las variaciones a través del día y la noche en magnitud de estas variables, exponen a los semovientes a periodos máximos o mínimos de exposición por periodos variables, que podrían alteran la zona de termo neutralidad de los animales (Cerqueira et al., 2016). En general, se estima que cuando la temperatura máxima supera los $27{ }^{\circ} \mathrm{C}$ el ambiente es estresante para los animales (Cony et al., 2004). Excesos de precipitación podrían generar saturación del suelo, un mayor pisoteo sobre la pastura, una menor sensación térmica por los animales, situación que, a largo plazo, afecta la disponibilidad de forraje y, también los animales podrían destinar nutrimentos para calentarse (WingChing-Jones et al., 2008). Por ejemplo, conforme aumentó la radiación registrada, se determinó una tendencia a disminuir la producción de leche en el sistema a $1800 \mathrm{msnm}$, lo que indica que una mayor radiación se asocia con menor nubosidad, lo que podría provocar que en horas de la noche se registren bajas temperaturas y menor precipitación, efectos que generan una utilización de los nutrimentos para calentamiento, o que los animales se agrupen para mantener el calor, por lo que el consumo de materia seca disminuye. De igual manera, durante el día valores altos de radiación inciden en un aumento del calor del semoviente lo que afecta de forma negativa el consumo de forraje.

\section{Conclusiones}

El conocer las condiciones climáticas donde se desarrolla la actividad productiva, permite asociar las variaciones del clima con la productividad del hato, relación que posibilita a los responsables de los animales tomar medidas correctivas para mantenerlos en la zona de termo neutralidad.

Valores de IHT de 56 y 65 provocaron mermas en la producción láctea de vacas jersey ubicadas a 1800 y 600 $\mathrm{msnm}$, respectivamente; lo que indica que estas presentaron estrés por frío o calor.

Es importante considerar las variaciones a través del día y la noche en magnitud de la temperatura, precipitación, radiación y humedad relativa, debido a que exponen al ganado a periodos variables de máximos o mínimos, que podrían alteran la zona de termo neutralidad de los animales.

La generación de indicadores productivos que se obtienen al analizar la producción láctea durante lactancias sucesivas o históricas del sistema de producción, permite establecer una herramienta de selección de reemplazos, con la intención de superar anualmente los promedios de producción, al escoger animales que se encuentran por encima del promedio de producción, como también establecer un tiempo máximo de permanencia de un animal como unidad productora eficiente.

\section{Agradecimientos}

Se agradece el apoyo del Ing. José Joaquín Jiménez Zamora en el desarrollo de esta investigación, y a los señores Roberto Ugalde y Oscar Garita, por la dedicación en sus funciones en el Módulo Lechero de la Universidad de Costa Rica. 


\section{Literatura citada}

Alcina, A., C. Forfan, E. Valencia, y J. Yokohama. 2001. Condición ambiental y su efecto en la temperatura rectal y frecuencia respiratoria en bovinos cruzados (Bos taurus X Bos indicus) del estado de Yucatán, México. Rev. Biomed. 12:112-121. doi:10.32776/revbiomed.v12i2.263

Arias, R., T. Mader, y R. Escobar. 2008. Factores climáticos que afectan el desempeño productivo del ganado bovino de carne y leche. Arch. Med. Vet. 40:7-22. doi:10.4067/S0301-732X2008000100002

Berman, A. 2011. Invited review: Are adaptations present to support dairy cattle in warm climates. J. Dairy Sci. 94:2147-2158. doi:10.3168/jds.2010-3962

Carvajal, M., E. Valencia, y J. Segura. 2002. Duración de la lactancia y producción de leche en el estado de Yucatán, México. Rev. Biomed 13:25-31. doi:10.32776/revbiomed.v13i1.292

Cerqueira, J.O.L., J.P.P. Araújo, I. Blanco-Penedo, J. Cantalapiedra, A.M.D. Silvestre, y S.J.C.R. Silva. 2016. Predicción de estrés térmico en vacas lecheras mediante inidcadores ambientales y fisiológicos. Arch. Zootec. 65:357-364. doi:10.21071/ az.v65i251.697

Collier, R.J., C. Coppola, and A. Wolfgram. 2003. Novel approaches for the alleviation of climatic stress in farm animals. Interactions between Climate and Animal Production. EAAP Techn. Ser. 7:61-72.

Collier, R., G. Dahl, and J. Van-Baale. 2006. Major advances associated with environmental effects on dairy cattle. J. Dairy Sci.89:1244-1253. doi:10.3168/jds.S0022-0302(06)72193-2

Cony, P., G.A. Casagrande, y G.T. Vergara. 2004. Cuantificación de un índice de estrés calórico para vacas lecheras en Anguil, provincia de La Pampa (Argentina). Rev. Fac. Agron. 15(1/2):9-15.

Dikmen, S., and P.J. Hansen. 2009. Is the temperature-humidity index the best indicator of heat stress in lacting dairy cows in a subtropical environment? J. Dairy Sci. 92:109-116. doi:10.3168/jds.2008-1370

Haworth, G.M., W.P. Tranter, J.N. Chuck, Z. Cheng, and D.C. Wathes. 2008. Relationships between age at first calving and first lactation milk yield, and lifetime productivity and longevity in dairy cows. Vet. Record. 162:643-647. doi:10.1136/ vr.162.20.643.

INEC (Instituto Nacional de Estadísticas y Censos). 2015. VI Censo Nacional Agropecuario: Resultados generales. INEC, San José, CRI.

IMN (Instituto Metereológico Nacional). 2008. El clima, su variabilidad y cambio climático en Costa Rica. Cambio Climático/ Segunda comunicación Nacional. IMN, San José, CRI.

IMN (Instituto Metereológico Nacional). 2015. Clima en Costa Rica. El clima y las regiones climáticas de Costa Rica. IMN, San José, CRI. https://www.imn.ac.cr/documents/10179/31165/clima-regiones-climat.pdf/cb3b55c3-f358-495a-b66c90e677e35f57 (consultado 15 may. 2015).

Jiménez, A.E., V.M. Montenegro, J. Hernández, G. Dolz, L. Maranda, J. Galindo, C. Epe, and T. Schnieder. 2007. Dynamics of infections with gastrointestinal parasites and Dictyocaulus viviparus in dairy and beef cattle from Costa Rica. Vet. Parasitol. 148:262-271. doi:10.1016/j.vetpar.2007.06.015

Jordan, E. 2003. Effects of heat stress on reproduction. J. Dairy Sci. 86:(Suppl.):E104-E114. doi:10.3168/jds.S00220302(03)74043-0

Kadzere, C.T., M.R. Murphy, N. Silanikove, and E. Maltz. 2002. Heat stress in lactating dairy cows: a review. Livest. Prod. Sci. 77:59-91. doi:10.1016/S0301-6226(01)00330-X 
Khalifa, H.H. 2003. Bioclimatology and adaptation of farm animals in a changing climate. In: N. Lacetera et al., editors, Interactions between climate and animal production. Wageningen Academic Publishers, Wageningen, NLD. p. 15-29.

Lucy, M.C. 2001. Reproductive loss in high-producing dairy cattle: Where will it end? J. Dairy Sci. 84:1277-1293. doi:10.3168/ jds.S0022-0302(01)70158-0

Martínez, A. 2011. Estrés calórico en el ganado lechero (Bos taurus) de la raza Holstein. Tesis M.Sc., Universidad Autónoma de Querétaro, MEX.

MINAET/IMN/PNUD/CRRH (Ministerio del Ambiente Energía y Telecomunicaciones/Instituto Meteorológico Nacional/ Programa de las Naciones Unidad para el Desarrollo/Comité Regional de Recursos Hidráulicos). 2008. El clima, su variabilidad y cambio climático en Costa Rica. Segunda comunicación nacional. University of Florida, FL, USA. http://users.clas.ufl.edu/prwaylen/geo3280articles/el_clima_variabilidad_y_cambio_climatico_en_cr_version_final.pdf (consultado 3 jul. 2018).

NRC (National Research Council). 2001. Nutrient requirements of dairy cattle. 7th ed. National Academy Press, WA, USA.

Pérez, G., y M. Gómez. 2005. Factores genéticos y ambientales que afectan el comportamiento productivo de un rebaño pardo suizo en el trópico. 1. Producción de leche. Rev. Científ. FCV-LUZ 15(2):141-147.

Pezzopane, J.R.M., P.M. Santos, S.R.M. Evangelista, C. Bosi, A.C.R. Cavalcantes, G.M. Bettiol, C.A. de-Miranda, and G.Q. Pellegrino. 2016. Panicum maximum cv. Tanzania: climate trends and regional pasture production in Brazil. Grass Forage Sci. doi:10.1111/gfs.12229

Polsky, L., and A. von-Keyserlingk. 2017. Invited review: Effects of heat stress on dairy welfare. J. Dairy Sci. 100:8645-12651. doi:10.3168/jds.2017-12651

Quesada, R. 2007. Los bosques de Costa Rica. IX Congreso Nacional de Ciencias. Instituto Tecnológico de Costa Rica. http:// www.cientec.or.cr/exploraciones/ponencias2007/RupertoQuesada.pdf (consultado 03 mar.2017).

Quintero, B., T. Clavero, C. Castro, A. del-Villar, y O. Araujo. 1995. Efectos de los factores climáticos y altura de corte sobre el valor nutritivo y producción de materia seca del pasto elefante enano (Pennisetum purpureum Schum. cv. Mott). Rev. Fac. Agron. (Luz) 12:81-94.

Retana J.A. 2015. El clima y la ganadería bovina en Costa Rica. CIENTEC, San José, CRI. https://www.imn.ac.cr/ documents/10179/20909/Clima+y+producci\%C3\%B3n+ganadera (consultado 10 jul. 2018).

Rodríguez, D., H. Amores, J. Murillo, O.M. Bogantes, R. Barrantes, O. Arguedas, M. Arrieta, W. Mora, y A. Vargas. 2007. Agrocadena de leche. Ministerio de Agricultura y Ganadería, CRI. http://www.mag.go.cr/bibliotecavirtual/E70-10453. pdf (consultado 10 jul. 2018).

SAS Institute Inc. 2003. SAS 9.1.3 for Windows. Service Pack 4. Win Pro plataforma. Copyright (c) 2002-2003. SAS Institute Inc., Cary, NC, USA.

Sánchez, J., y H. Soto. 1999. Estimación de la calidad nutricional de los forrajes del cantón de San Carlos. III Energía para la producción de energía. Nutr. Anim. Trop. 5(1):31-49.

Sánchez, J., M.Villareal, y H. Soto. 2000. Caracterización nutricional de los componentes forrajeros de cuatro asociaciones gramíneas/Arachis pintoi. Nutr. Anim. Trop. 6(1):1-22.

Silanikove, N. 2000. Effects of heat stress on the welfare of extensively managed domestic ruminants. Livest. Prod. Sci. 67:118. doi:10.1016/S0301-6226(00)00162-7

Snyder, R., P. de-Melo-Abreu, y S. Matulich. 2010. Protección contra las heladas: fundamentos, práctica y economía. Vol. 2. Serie sobre el medio ambiente y la gestión de los recursos naturales. FAO, Roma, ITA. 
Soydan, E., and M. Kuran. 2017. The effect of calving season on reproductive performance of jersey cows. Mljekarstvo 67(4):297-304. doi:10.15567/mljekarstvo.2017.0407

Stadnik, L., S. Atasever, and J. Duchácek. 2017. Effects of body condition score and daily milk yield on reproduction traits of czechfleckvieh cows. Anim. Reprod. 14:1264-1269. doi:10.21451/1984-3143-AR944

Staples, C.R., and W.W. Thatcher. 2011. Stress in dairy animals/ Heat stress: Effects on milk production and composition. In: J.W. Fuquay, Encyclopedia of dairy sciences. 2nd ed. Academic press, Cambridge, MA, USA. p. 561-566. doi:1016/ B978-0-12-374407-4.00467-2

Vargas, G. 2006. Geografía de Costa Rica. EUNED, San José, CRI.

Van-Soest, P.J. 1994 Nutritional ecology of the ruminant. 2nd ed. Cornell University Press, Ithaca, NY, USA.

West, J. 2003. Effects of heat-stress on production in dairy cattle. J. Dairy Sci. 86:2131-2144. doi:10.3168/jds.S00220302(03)73803-X

WingChing-Jones, R., R. Pérez, y E. Salazar. 2008. Condiciones ambientales y producción de leche de un hato de ganado Jersey en el trópico Húmedo: El caso del Módulo Lechero-SDA/UCR. Agron. Costarricense 32(1):87-94.

Zúñiga, R., L. Figueroa, L. Ruiz., J. Murillo, L. Obando, A. Del-Pino, F. Murillo, L. Morales, y D. Alvarado. 2005. Guía estratégica de acción para la Cámara Nacional de Productores de Leche de Costa Rica. INCAE, CRI. 